\title{
Therapeutic Misconception in Psychiatry Research: A Systematic Review
}

\author{
Ivan SK Thong ${ }^{1}$, Meng Yee Foo', Min Yi Sum², Benjamin Capps ${ }^{3}$, Tih-Shih Lee ${ }^{4}$, Calvin $\mathrm{Ho}^{5}$, Kang Sim²,6 \\ ${ }^{1}$ The College of Arts and Sciences, University at Buffalo, Buffalo, New York, USA, ${ }^{2}$ Research Division, Institute of Mental Health, Singapore, \\ Singapore, ${ }^{3}$ Department of Bioethics, Faculty of Medicine, Dalhousie University, Halifax, Nova Scotia, Canada, ${ }^{4}$ Duke-NUS Graduate \\ Medical School, ${ }^{5}$ Centre for Biomedical Ethics, Yong Loo Lin School of Medicine, National University of Singapore, ${ }^{6}$ Department of General \\ Psychiatry, Institute of Mental Health, Singapore, Singapore
}

\begin{abstract}
Therapeutic misconception (TM) denotes the phenomenon in which research subjects conflate research purpose, protocols and procedures with clinical treatment. We examined the prevalence, contributory factors, clinical associations, impact, and collated solutions on TM within psychiatric research, and made suggestions going ahead. Literature search for relevant empirical research papers was conducted until February 2015. Eighty-eight reports were extracted, of which 31 were selected, summarised into different headings for discussion of implications and collated solutions of TM. We found variable and high rates of TM (ranging from $12.5 \%$ to $86 \%$ ) in some psychiatry research populations. Contributory factors to TM included perceived medical roles of researchers, media, research setting and subject factors. Greater TM in affective, neurodevelopmental and psychotic spectrum conditions were associated with demographic variables (such as lower education, increased age), clinical factors (such as poor insight, cognitive deficits, increased symptoms, poorer self-rated quality of health), and social functioning (such as decreased independence). Inattention to TM may lead to frustration, negative impression and abandonment of participation in psychiatry research. Strategies such as the employment of a neutral educator during the informed consent process and education modules may be effective in addressing TM. Further research is warranted to examine the different TM facets, specific clinical correlates and more effective management strategies.
\end{abstract}

KEY WORDS: Therapeutic misconception; Psychiatry; Research; Affective disorders, psychotic.

\section{INTRODUCTION}

The term therapeutic misconception (TM) was first coined by Paul Appelbaum who found that patients with psychiatric conditions could conflate imperatives of research with treatment aims. ${ }^{1,2)}$ In an earlier Hasting Centre report, ${ }^{3)} \mathrm{TM}$ is considered a side effect of powerful scientific methods like randomised controlled trials (RCT) which are gold standards used in the investigations of the efficacy of different medical interventions, and hence TM must be managed accordingly. Attributing value to the principle of autonomy and promoting trust and cooperation may require researchers to explain to research participants who are unable to deal with TM why it might not even be in their best interests to participate in the study. ${ }^{3)}$ Over time, there has been some further elaboration on the

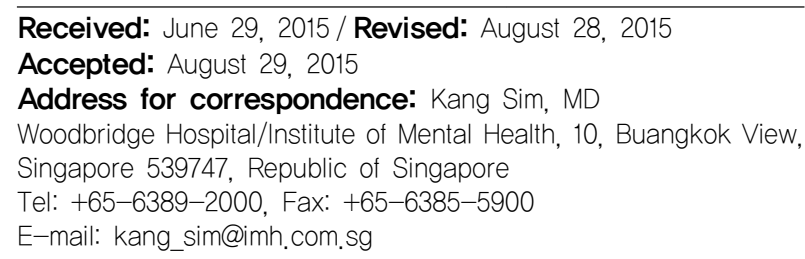

various dimensions that TM encompasses. ${ }^{4-8)}$ Initially, TM was proposed as a term to indicate the general concept of conflating research and treatment imperatives. ${ }^{3)}$ Subsequently, the concept of TM has been further elaborated to include different combinations of incorrect expectation of individualized care (TM1) and mis-calculating risk-benefit ratio (TM2), ${ }^{9}$ as well as suggested domains of perceived dual clinician- roles, adherence to strict protocol, ${ }^{4)}$ and the goals of the research (TM3), which expand the comparability of findings across studies, examination of prevalence of TM across fields as well as interventions to reduce it. ${ }^{4,7)}$

In view of the significance of TM in clinical research, we sought to examine the existing literature on TM, defined in the three main dimensions of incorrect expectation of individualised care (TM1), incorrect assessment of benefits and risks (TM2), and incorrect understanding of research goals (TM3) ${ }^{1)}$ within research in psychiatric populations, and their prevalence, associated contributory factors, clinical associations, impact, and proposed solutions. In the process, we also discussed about the limitations of past research and make suggestions to move for-

(a) This is an Open-Access article distributed under the terms of the Creative Commons Attribution Non-Commercial License (http://creativecommons.org/licenses/by-nc/4.0) which permits unrestricted non-commercial use, distribution, and reproduction in any medium, provided the original work is properly cited. 
ward with research on understanding and alleviating TM.

\section{METHODS}

\section{Literature Search}

A literature search was conducted using two major databases, the National Centre for Biotechnology Information (NCBI) PubMed/Medline and Web of Science, to identify published empirical research papers from 1982 when the term TM was first coined until February 2015 which investigated TM within psychiatric research. The keywords used included "therapeutic misconception", "psychiatric", "mental disorders", "research". All potential papers of interest were retrieved and evaluated for satisfaction of inclusion criteria described below. Additionally, references from selected articles were also evaluated and additional articles were included if they met the inclusion criteria.

\section{Inclusion Criteria}

The articles were included in the review if they satisfied the following criteria: (1) the article was published in peer-reviewed journals in English, (2) the empirical study addressed issues of TM involving psychiatric subjects. Abstracts, conference proceedings, review and opinion papers were excluded.

\section{Data Extraction and Synthesis}

The process involved familiarization with the data from the reviewed studies, generating initial codes for issues, searching for main issues, reviewing the issues, defining and naming the issues and then collating the findings. Specifically, we summarised the extant data into headings of 1) prevalence, 2) contributory factors, 3) clinical associations, 4) consequences, and then discuss the implications and collated solutions of TM.

\section{RESULTS}

The search process considered a total of 88 potentially useful reports, of which 31 satisfied entry criteria and provided data are reported here.

\section{Dimensions of TM in Psychiatry Research}

The three dimensions of TM are as follows:

a) TM1: incorrect expectation of individualised care ${ }^{1,2,9-18)}$

b) TM2: incorrect assessment of benefits and risks (also known as Therapeutic Mis-estimation), ${ }^{13)}$ which relates to unreasonable evaluation of likelihood or na- ture of benefit, 1,9,10,12,18,19) and/or under-appreciation of risks that stem from research participation ${ }^{3,14,20)}$

c) TM3: incorrect understanding of the goals of the study ${ }^{1,12)}$ involving thinking that research is primarily to help participants rather than to generate scientific knowledge (1-23,17)

\section{Prevalence}

TM's prevalence was first found in patients with schizophrenia and borderline personality disorders ${ }^{2}$ and later extended to those subjects with depression. ${ }^{3)}$ According to a study involving subjects with depression or attention deficit hyperactivity disorder, $61.8 \%$ was found to manifest TM1, TM2, or both. ${ }^{9)}$ In another study involving patients with affective and other disorders, up to $86.5 \%$ of subjects were unable to cite any risk or disadvantages concerning the research design which involved randomisation and use of placebos. ${ }^{14)}$ Dunn et al. ${ }^{11)}$ found that up to $69 \%$ of participants with schizophrenia had TM1. However, in another study of TM involving schizophrenics engaged in an actual clinical trial, TM 1 was not prominent and there was no prominent worsening in TM over the course of 8 weeks. ${ }^{21)}$ TM was also not found to be prominent in parents consenting for research on children with autism in that $72 \%$ of parents knew that treatment assignment would not be based on their child's individual needs, and over $90 \%$ of them knew that a double blind would be employed, that their child could be assigned to placebo, were aware of the probability of receiving a placebo, and correctly stated that the primary purpose of the study was to test medication efficacy. ${ }^{16)}$ More parents of children with autism (72\%) understood the concept of randomisation compared to parents of children with leukaemia $(50 \%) .{ }^{24)}$ Of note, the prevalence of TM3 concerning purpose of research stood at $12.5 \%$ to $15 \%$, ${ }^{1,7)}$ which was lower compared to the other two TM dimensions.

\section{Contributory Factors towards the Development of TM}

Belkin $^{25)}$ argues for the importance of taking into account social, historical and other contexts in order to correctly understand and apply ethical requirements. Under certain conditions, it may not be realistic to conduct research that is entirely free of TM. Hence, misunderstanding or misapplying ethical requirements could give undue emphasis to TM, and so hinder research. This makes TM a major concern for research in psychiatry. In addition, the media has been found to influence participants into seeking out research in the hope of receiving better treatment ${ }^{26-28)}$ such as in the case of research on deep brain 
stimulation. ${ }^{29,30)}$ Media has also influenced participants to think that the goal of a research study is to improve the participant's outcome. ${ }^{31)}$ In the process, participants become unable to see how research and treatment goals are incompatible. $^{2)}$

Expectation of individualized care could have arisen from the clinical duties of physicians who are also researchers, ${ }^{3,5,15,32)}$ who simultaneously recruit their patients into trials. ${ }^{33,34)}$ In addition, research is also almost exclusively conducted in clinical settings ${ }^{18)}$ where standard treatment is normally provided. ${ }^{35)}$ Researchers don white coats, perform research with the same equipment used in standard care $^{35)}$ and perform procedures (such as venepunctures, electrocardiograms) that are similar to those performed in treatment. ${ }^{6)}$ Therapeutic terminology is also present in consent documents. ${ }^{36)}$ Thus, it is not surprising that participants see research as an extension of their usual treatment. ${ }^{33)}$ In some situations, the desperation experienced by subjects and caregivers may compel them to believe that research is beneficial, ${ }^{9,19)}$ thus driving them to research participation even if the possibility of benefit is low, ${ }^{26,37-39)}$ suggesting that situational conditions may accentuate specific dimensions of TM.

Within the session for explanation about the study, the inner motivation for better treatment driving some subjects to participate may result in the shift of mind frame from one of giving consent for research to one of receiving individual care in the course of research. The mind frame held during these sessions is important as it may create expectations that guide interpretations of what is being said. ${ }^{2,34)}$ For example, certain aspects of research necessary for ensuring safety and wellbeing of research subjects, such as monitoring for adverse occurrences, may be mistaken as proof of individualised care. ${ }^{40}$

Furthermore, participants have a tendency to entrust their wellbeing to their physicians ${ }^{3,41)}$ and researchers, ${ }^{42)}$ with the assumption that they will look after their best clinical interest, ${ }^{42)}$ and that physicians would recommend research for their benefit ${ }^{9,10,43,44)}$ without exposing them to significant risks. ${ }^{37)}$

\section{Clinical Associations}

Most of the extant studies examined the issue of TM within affective spectrum conditions, neurodevelopmental disorders and psychotic spectrum conditions. Overall, dimensions TM1, TM2, and TM3 were found to be strongly correlated with one another. ${ }^{1)}$

\section{Affective spectrum conditions}

TM1 and TM2 combined were associated with greater age, optimism about their condition in 6 months, lower education, optimism about current health condition, when there was risk of death from intervention, and when there were US Food and Drug Administration approved medication available." TM1 and TM2 combined were also associated with more impaired physical functioning, physical role limitations, vitality, social functioning, general mental health and emotional role limitations as measured by the Short Form (36) Health Survey (SF-36) scale.") Lidz et al. ${ }^{14)}$ found that middle aged subjects and females were more likely to recognize risks related to research design. In general, the results suggest that worse self-reported health status is associated with higher probability of having TM1 and TM2. In a study of patients with treatment-resistant depression, depression severity correlated negatively with TM1, TM2, and TM3 combined. ${ }^{12)}$ Despite good decisional capacity as measured by the MacArthur Competence Assessment Tool for Clinical Research (MacCat-CR) Understanding, Reasoning, and Appreciation Scale, subscales, TM1, TM2, and TM3 combined were still prevalent among participants with difficult to treat depression. ${ }^{12)}$ The TM ratings on the risks of electroencephalography and magnetic resonance imaging had negative correlations with depression scores, suggesting greater understanding of the practical realities of research when depressed (depressive realism). ${ }^{12)}$

\section{Neurodevelopmental conditions}

Up to $18 \%$ of participants with mild mental retardation and $4 \%$ of participants with moderate mental retardation were able to appreciate the purpose of research. ${ }^{45)}$ Participants with intellectual disability were less able to understand the concepts of randomization, placebo, and purpose of research according to the Assessment of Consent Capacity-Randomized Clinical Trials (ACC-RCT). ${ }^{45}$ ) In a study involving parents of patients with autism, TM1 was found to be associated with lower levels of education, eg parents with a college degree were significantly more likely than parents without a college degree to indicate that the allocation of treatment for their autistic children was done randomly. ${ }^{16}$ Of note, misunderstanding research assignment as individualized clinical care amongst those with pre-existing therapeutic relationship with clinician researcher $(14 \%)$ was not greater compared with overall sample including those with no prior therapeutic relationship (27\%). ${ }^{16)}$ 


\section{Psychotic spectrum conditions}

TM1 was found to be negatively correlated with education, Birchwood Insight Questionnaire score, decisional capacity as measured by MacCAT-CR subscales, and positively with cognitive deficits as measured by the Mattis Dementia Rating score in patients with schizophrenia and schizoaffective disorder. ${ }^{11)}$ Participants exhibited less TM when living in an apartment or house themselves than living in residential facilities, indicating association of TM with poorer independence in activities of daily functioning. ${ }^{11)}$ Of note, TM1 in patients with schizophrenia patients was positively correlated with Positive and Negative Syndrome Scale (PANSS) positive and general subscale scores, but had no association with MacCat-CR appreciation and reasoning subscales. ${ }^{11)}$ A separate study examining the understanding of placebo found that in patients with schizophrenia, worse cognitive deficits and worse negative psychotic symptom scores were associated with TM2, indicating miscalculation of benefit risk ratio. $^{20)}$

\section{Consequences}

Understanding the impact of TM on research participation is crucial. If there is incorrect understanding about the research process including the nature and aims of the project, benefits and risks evaluation, ${ }^{15,32,46)}$ a participant may be consenting to something different from what the study actually covers. ${ }^{19)}$ The question can then be asked of whether participants would have participated if they were corrected about their understanding of the research details. ${ }^{46)}$ For studies that require a considerable period of follow up, the presence of TM may result in subjects continuing with enrolment even if it may be burdensome to them. ${ }^{18)}$ If participants only realise halfway that individualised care is not apparent, benefits unforthcoming or research aims without therapeutic purpose, they might decide to drop out of the study and disrupt the research process. $^{15)}$

The presence of TM1 may compromise informed consent as participants were less aware of the limitations on individualized care from research design. ${ }^{14)}$ Unattended, TM1 can result in frustration within the participants when true appreciation of the nature of the study occurs later in the study. Regarding TM2 and TM3, if participants were to realise it after the study is complete, it may result in a negative impression about the study team, research process, and subsequent unwillingness to participate in future research. ${ }^{15,47)}$

\section{DISCUSSION}

This systematic review of extant literature pertaining to TM in psychiatry research revealed several main findings. First, we found considerably high rates of TM, especially TM1 (up to 69\%) and TM2 (up to $86 \%$ ), in psychiatry research studies compared with TM3 (up to 15\%). Second, contributory factors to TM included perceived medical roles of researchers, media, research setting and subject factors. Third, greater TM in affective, neurodevelopmental and psychotic spectrum conditions were associated with demographic variables (such as lower education, increased age), clinical factors (such as poor insight, cognitive deficits, increased symptoms, poorer self-rated quality of health), functioning (such as decreased independence). Inattention to TM may lead to frustration and negative impression of the framework in psychiatry research.

Whether TM is considered present in a patient can only be assessed within the framework of a particular proto$\mathrm{col}^{33)}$ and may not be entirely removable. To assess TM accurately, treatment in research trials needs to be juxtaposed against available health care alternatives as it is probably not unreasonable for participants to seek benefit from research in situations where alternative high quality care is not accessible, ${ }^{48)}$ when trials introduce rare lifesaving opportunities, ${ }^{49)}$ or when trials provide some of the best-quality care otherwise unobtainable elsewhere. Subjects in a study testing methamphetamine dependency treatment were probably justified in seeking clinical trials as a treatment option, as standard pharmaceutical treatments continue to be researched and developed ${ }^{50)}$ and likewise for human immunodeficiency virus infection and acquired immune deficiency syndrome (HIV/AIDS) patients participating in research in the 1980s where RCT was one of the only treatment options available. ${ }^{51)} \mathrm{TM}$ is difficult to remove because correcting erroneous beliefs about research purpose and benefits risk paternalistically enforcing notions of research on research subjects. ${ }^{46)}$ Excluding participants based on insufficient understanding of research details also reduces onus of better explanation by research teams in the course of their research. ${ }^{46)}$ Moreover, it may be impractical to expect people to agree to research participation singularly without any hope, desire or expectation of therapeutic benefit in the decision-making process. ${ }^{52}$

Regarding TM1, understanding of the scientific enterprise did not necessarily equate to participants being able 
to apply the knowledge to their own. Appelbaum et al. ${ }^{2)}$ reported about a participant who was aware that assignment to treatment conditions would be random, but hesitated before stating erroneously that assignment would be based on individual needs when questioned on how it would be applied specifically to her. This shows that appreciation and application of research design to the subject is not assured even when understanding of research design is present. ${ }^{2)}$ Although the aforementioned participant was considered to manifest TM by Appelbaum et al., ${ }^{2)}$ Swekoski and Barnbaum ${ }^{53)}$ argued to the contrary as the participant clearly understood the nature of research and the belief for individualized care did not stem from a misconception regarding the nature of research but rather "unrealistic optimism". Since expectation of individualized care (TM1) has been shown to result from causes other than conflation of research and treatment imperatives, ${ }^{53)}$ more study is needed to clarify the factors that may account for participant's belief in individualised care during research.

Concerning TM2, Horng and Grady ${ }^{13)}$ renamed TM2 as "therapeutic mis-estimation" as it involves misunderstanding of probability of benefits and risks rather than the more general misunderstanding about the nature and intent of research. In their framework, therapeutic mis-estimation can supposedly exist alongside an accurate understanding of the nature and purpose of research. ${ }^{4,13)}$ Appelbaum et al. ${ }^{3)}$ stated that failure to understand research methods may result in mis-estimating the risk-benefit ratio of the protocol within the research subjects ${ }^{3)}$ suggesting that TM2 may be a consequence of other dimensions of TM such as TM1 and TM3 ${ }^{54)}$ Miller and Joffe ${ }^{8)}$ held a similar view stating that TM1 captures the core concept of conflating research with treatment but it is not so clear if TM2 does the same. Of note, therapeutic mis-estimation is distinct from therapeutic optimism which refers to the research subjects hoping for the best outcome in the course of participation in research. ${ }^{13)}$ In a study consisting of participants with Parkinson's disorder, 50\% of subjects desired therapeutic benefit from participation in a phase 1 gene transfer trial. Upon detailed questioning, however, it revealed that participants were being optimistic rather than misled. They saw their decision to participate in the specific research study as a 'gamble' and showed awareness that there were no misguided promises of therapeutic benefit or favourable odds of benefits over risks. ${ }^{7)}$ This highlights how therapeutic optimism could also have been mistaken as TM2, when the former may be considered as ethically less problematic. ${ }^{13)}$
With regard to TM3, participants may explicitly express an incorrect understanding of the detailed purpose of a trial but may be concurrently aware of its broad scientific objectives. Kim et al. ${ }^{7)}$ found in their study that half of the participants with Parkinson's disease stated incorrectly the detailed purpose of the research at the beginning but, upon further probing, it was revealed that they understood that the broad objective of the study was to help future patients with a similar condition. In all research areas including psychiatry, current research activities may not always produce results that directly benefit current subjects. ${ }^{4}$ Kimmelman $^{54)}$ argues that research can have a plurality of purposes as it should attend to the interests of its users, and that multiple objectives can be achieved including that of helping to manage the condition of the current subjects and yet potentially produce future benefits.

Furthermore, the dual role of clinicians to provide specific care and yet probe for generalizable data from their research involving the same subjects is considered by some to be incompatible. ${ }^{55)}$ Studies have examined the prevalence of TM amongst researchers although not within the arena of psychiatry research. More than a third (38.2\%) of paediatric oncologists, $19.5 \%$ of medical oncologists, and $21 \%$ of other oncology specialists reported that the main purpose of clinical trials is to ensure that participants receive state-of-the-art treatment rather than to improve treatment for future cancer patients. ${ }^{23)}$ Also, in an early-phase gene transfer research, $46 \%$ of researchers expected their participants to receive direct therapeutic benefit. $^{56)}$

Researchers who conflate research with treatment would be unlikely to provide accurate information to better address the issue of TM in participants. ${ }^{6}$ ) This notion is supported by findings from a study ${ }^{23)}$ which surveyed the reasons of oncologists enrolling patients into clinical trials. It was found that a significant proportion of oncologists (more than $50 \%$ of medical oncologist, $60 \%$ of paediatric oncologists, and $55 \%$ of other subspecialist oncologist respectively) reported that the enrolment of their patients was to receive state of the art treatment. ${ }^{23)}$ Specific study information proffered to participants has been identified as an important determinant of participants' understanding of the nature and purpose of the research study, elements which are examined in TM. ${ }^{57-59)}$ Adolescents and parents were also found to express high regard for the advice of physicians regarding trial, ${ }^{41)}$ with many physicians doubling as researchers when they recruit their own patients into the trials. ${ }^{33)}$ As a result, researcher TM may blind researchers to the risks that partic- 
ipants are being exposed in the course of research ${ }^{10)}$ and one may argue whether this is one of the predisposing factors which contributed towards past infamous incidents in the history of research ethics. ${ }^{60-63)}$

Pertaining to TM "at risk" populations, patients who are unemployed and with limited financial support may be more susceptible to TM as they seek mental health care, ${ }^{64)}$ especially with regard to early phase research where chances of direct benefit are less clear. ${ }^{65)}$ In a similar vein, TM is thought to be more prevalent in participants involved in phase 1 clinical trials, as opposed to later-phase trials, ${ }^{7)}$ because therapeutic benefits expected by subjects in the earlier phase trials are less certain, hence the conduct of such studies to clarify the risk benefit evaluation. Phase 1 trials are focused on investigating the dose range and safety of a test drug, ${ }^{65)}$ but participants may be compelled to participate because of fear and desperation about the efficacy of existing treatment options. ${ }^{52)}$ Thus, researchers should be careful to consider TM in the context of other factors such as access to healthcare, socio-economic factors and type of trials. ${ }^{54,64)}$

\section{Collated Solutions}

Inadequate understanding of research methodologies can be of concern, especially if this results in unawareness of how these procedures are or aren't included in the present clinical management. ${ }^{3,9,65)}$ As such, some suggestions within the existing literature related to ways to improve the understanding of research procedures within research subjects.

Personal sessions with neutral staff during informed consent appear to be an effective measure against TM. ${ }^{3,58)}$ Within a group of patients with schizophrenia, borderline personality disorder and depression, and who underwent such personal sessions, $50 \%$ understood that randomization would be used compared to $25 \%$ in the control group, $100 \%$ understood that placebo would be employed compared to $73 \%$ in the control group, $56 \%$ understood that double blind would be used compared with $31 \%$ in the control group, and $29 \%$ recognized limits on their treatment due to constraints of the protocol compared with $2 \%$ in control group. ${ }^{3)}$ A similar finding was noted in another study consisting of participants from the same populations in which the use of a neutral educator significantly improved the understanding of research purpose and randomization when compared to the standard procedure. ${ }^{58)}$ In a more recent study of older schizophrenia patients, employing an education module on the concept of placebo significantly improved subjects' understanding of it. ${ }^{11)}$
These findings suggest that whilst better understanding of research methodologies may not completely reverse $\mathrm{TM},{ }^{2)}$ it can be an important step to educate and reduce TM. $^{11)}$

Institutional review boards have been found to play a role in reducing TM in participants via a few aspects. This can occur through several ways, such as enforcing the aforementioned neutral assessors or educators, ensuring clarification of informed consent forms in delineating research and treatment procedures, as well as enforcing clarity of the risks and benefits of the research, mental capacity assessments for consent, and continual assessment of consent, especially in the background of vulnerable and cognitively impaired subjects. ${ }^{66-68)}$

During disclosures about research, researchers should try to maintain an informed consent frame instead of a clinical frame ${ }^{34)}$ through a review of the covered content in the consent documents. ${ }^{15)}$ Also, some authors have suggested using layman terms to explain research procedures $^{3,15)}$ as research subjects may lack understanding of technical jargons like placebo and randomization. ${ }^{69,70)}$ Misleading terms should be cautioned against such as using the term 'sugar pills' to describe placebo has led to schizophrenia patients mistaking placebo as a type of medication for treating diabetes. ${ }^{11)}$

While making conscious efforts to communicate using layman terms, researchers should highlight the distinction between routine clinical interventions and research on a new intervention. ${ }^{15)}$ The nature and goals of the research study, pertinent risks and benefit, and availability of same or different treatment options outside the study should also be mentioned, ${ }^{15)}$ together with the fact that the researcher would be unable to provide personal care within the specific context of the research study. ${ }^{2)}$ An addendum containing information of how procedures of research are not part of standard care should be regularly attached to consent documents. ${ }^{54}$

Additionally, the understanding of the participants should be assessed continually and noted and corrected for misconceptions as they occur. ${ }^{15)}$ However, if attempts to educate are unsuccessful, participants should be excluded from the study. ${ }^{46)}$ It has also been suggested that research staff involved in recruitment of subjects should be educated on TM as they have the opportunity and responsibility to communicate with patients and would be able to pick up on their misconceptions about research. ${ }^{71)}$ Of note, it is thought that monetary reimbursement might aid in reducing TM as it emphasizes the distinction of research from treatment. ${ }^{72)}$ Furthermore, focus groups could be 
used to generate discussion about beliefs regarding research to encourage in-depth thinking and increase awareness about the dimensions of TM. ${ }^{18)}$ Meurer et al. ${ }^{73)}$ proposed using "adaptive clinical trials" to reduce the disparity between participants' expectations of benefit and actual benefit received from research. This is done through an integration of new knowledge acquired over the course of the study into informed consent processes in order to improve the understanding of future participants. $^{73)}$

With regards to the assessment of TM2, it should be evaluated on the degree and personal significance of the mis-estimation of probabilities of benefits versus risks or level of therapeutic optimism as well. Participants should be presented with precise information about the probability and the extent of risk and benefit related to the research interventions. This presentation should be accompanied by alternatives within a predefined plan to manage TM. $^{13)}$ It has been suggested that qualitative methods should be employed to investigate how people resolve disparity between hope and desire for benefit and scientific goals of research so as to identify and manage therapeutic optimism if found. ${ }^{7)}$ The same should be employed in the assessment for TM3 within subjects to clarify whether they are aware that the primary purpose of research study is for advancement of knowledge for future treatment rather than immediate clinical management of the individual subjects. ${ }^{7)}$

\section{Limitations}

First, as studies of TM were mainly restricted to those consenting for research, the prevalence within populations without decision-making capacity is less clear. As less is known about how such misconceptions shape decision making in research participation, ${ }^{1)}$ this is even more difficult to assess in such populations with compromised decisional capacity. Second, methods and definitions used to assess TM were not standardized, possibly accounting for irregularities in the prevalence of reported TM across studies. ${ }^{1)}$ Third, it is difficult to examine the level of disclosure and the nature of information disclosed to participants within most studies which may further affect the prevalence of TM found. ${ }^{9}$ Fourth, participants have been found to concurrently possess accurate and contradictory understanding about research participation over time which may confound the study findings further, ${ }^{9)}$ warranting longitudinal studies of TM over time in psychiatric populations.

\section{CONCLUSIONS}

In conclusion, data regarding $\mathrm{TM}$ in psychiatry research is still relatively sparse. Nevertheless, TM seems prevalent amongst some psychiatric subjects, thus necessitating further research including prospective studies to examine their clinical correlates, impact as well as effective strategies to manage the different facets of TM. Future research should also focus on researchers of psychiatric populations, and clinicians referring cases for research studies as data suggest that TM in researchers might also affect potential participants in their understanding of the research protocol and procedures. ${ }^{6,23,56,74,75)}$ Use of standardized tools to assess TM should be encouraged (such as the TM scale by Appelbaum et al. ${ }^{1)}$ ) to allow comparability of results across studies and clinical research disciplines. More research is needed to investigate practical and effective measures and how they affect and influence decisions regarding research participation as towel as manage and reduce TM. ${ }^{10)}$

\section{REFERENCES}

1. Appelbaum PS, Anatchkova M, Albert K, Dunn LB, Lidz $\mathrm{CW}$. Therapeutic misconception in research subjects: development and validation of a measure. Clin Trials 2012; 9:748-761.

2. Appelbaum PS, Roth LH, Lidz C. The therapeutic misconception: informed consent in psychiatric research. Int $J$ Law Psychiatry 1982;5:319-329.

3. Appelbaum PS, Roth LH, Lidz CW, Benson P, Winslade W. False hopes and best data: consent to research and the therapeutic misconception. Hastings Cent Rep 1987;17:2024.

4. Henderson GE, Churchill LR, Davis AM, Easter MM, Grady C, Joffe S, et al. Clinical trials and medical care: defining the therapeutic misconception. PLoS Med 2007;4: e324.

5. Henderson GE, Easter MM, Zimmer C, King NM, Davis $\mathrm{AM}$, Rothschild BB, et al. Therapeutic misconception in early phase gene transfer trials. Soc Sci Med 2006;62: 239-253.

6. Goldberg DS. Eschewing definitions of the therapeutic misconception: a family resemblance analysis. $J$ Med Philos 2011;36:296-320.

7. Kim SY, Schrock L, Wilson RM, Frank SA, Holloway RG, Kieburtz K, et al. An approach to evaluating the therapeutic misconception. IRB 2009;31:7-14.

8. Miller FG, Joffe S. Evaluating the therapeutic misconception. Kennedy Inst Ethics $J$ 2006;16:353-366.

9. Appelbaum PS, Lidz CW, Grisso T. Therapeutic misconception in clinical research: frequency and risk factors. IRB 2004;26:1-8.

10. Appelbaum PS, Lidz CW. Twenty-five years of therapeutic misconception. Hastings Cent Rep 2008;38:5-6.

11. Dunn LB, Palmer BW, Keehan M, Jeste DV, Appelbaum PS. Assessment of therapeutic misconception in older schizophrenia patients with a brief instrument. Am J Psychiatry 
2006; 163:500-506.

12. Fisher CE, Dunn LB, Christopher PP, Holtzheimer PE, Leykin Y, Mayberg HS, et al. The ethics of research on deep brain stimulation for depression: decisional capacity and therapeutic misconception. Ann N Y Acad Sci 2012;1265: 69-79.

13. Horng S, Grady C. Misunderstanding in clinical research: distinguishing therapeutic misconception, therapeutic misestimation, and therapeutic optimism. IRB 2003;25:11-16.

14. Lidz CW, Appelbaum PS, Grisso T, Renaud M. Therapeutic misconception and the appreciation of risks in clinical trials. Soc Sci Med 2004;58:1689-1697.

15. Lidz CW, Appelbaum PS. The therapeutic misconception: problems and solutions. Med Care 2002;40(9 Suppl):V55V63.

16. Vitiello B, Aman MG, Scahill L, McCracken JT, McDougle CJ, Tierney E, et al. Research knowledge among parents of children participating in a randomized clinical trial. $J$ Am Acad Child Adolesc Psychiatry 2005;44:145-149.

17. Wasan AD, Taubenberger SP, Robinson WM. Reasons for participation in pain research: can they indicate a lack of informed consent? Pain Med 2009;10:111-119.

18. Wazaify M, Khalil SS, Silverman HJ. Expression of therapeutic misconception amongst Egyptians: a qualitative pilot study. BMC Med Ethics 2009;10:7.

19. Hoop JG, Smyth AC, Roberts LW. Ethical issues in psychiatric research on children and adolescents. Child Adolesc Psychiatr Clin N Am 2008;17:127-148.

20. Dunn LB, Palmer BW, Keehan M. Understanding of placebo controls among older people with schizophrenia. Schizophr Bull 2006;32:137-146.

21. Fischer BA, McMahon RP, Meyer WA, Slack DJ, Appelbaum PS, Carpenter WT. Participants with schizophrenia retain the information necessary for informed consent during clinical trials. J Clin Psychiatry 2013;74:622-627.

22. Joffe S, Weeks JC. Views of American oncologists about the purposes of clinical trials. J Natl Cancer Inst 2002;94:18471853.

23. Timmermans S, McKay T. Clinical trials as treatment option: bioethics and health care disparities in substance dependency. Soc Sci Med 2009;69:1784-1790.

24. Kodish E, Eder M, Noll RB, Ruccione K, Lange B, Angiolillo $\mathrm{A}$, et al. Communication of randomization in childhood leukemia trials. JAMA 2004;291:470-475.

25. Belkin GS. Misconceived bioethics?: The misconception of the "therapeutic misconception". Int J Law Psychiatry 2006;29:75-85.

26. Cassileth BR, Lusk EJ, Miller DS, Hurwitz S. Attitudes toward clinical trials among patients and the public. JAMA 1982;248:968-970.

27. Daugherty C, Ratain MJ, Grochowski E, Stocking C, Kodish E, Mick R, et al. Perceptions of cancer patients and their physicians involved in phase I trials. J Clin Oncol 1995;13: 1062-1072.

28. Gallo C, Perrone F, De Placido S, Giusti C. Informed versus randomised consent to clinical trials. Lancet 1995;346: 1060-1064.

29. Racine E, Waldman S, Palmour N, Risse D, Illes J. "Currents of hope": neurostimulation techniques in U.S. and U.K. print media. Camb Q Healthc Ethics 2007;16:312-316.

30. Skuban T, Hardenacke K, Woopen C, Kuhn J. Informed consent in deep brain stimulation - ethical considerations in a stress field of pride and prejudice. Front Integr Neurosci 2011;5:7.

31. Kasner SE, Baren JM, Le Roux PD, Nathanson PG, Lamond
$\mathrm{K}$, Rosenberg SL, et al. Community views on neurologic emergency treatment trials. Ann Emerg Med 2011;57:346354.

32. Appelbaum PS, Lidz C. The therapeutic misconception. In: Emanuel EJ, Grady C, Crouch RA, Lie RK, Miller FG, Wendler DD, editors. The Oxford textbook of clinical research ethics. New York: Oxford University Press;2008. p.633-644.

33. Brody $\mathrm{H}$, Miller FG. The clinician-investigator: unavoidable but manageable tension. Kennedy Inst Ethics $J$ 2003;13: 329-346.

34. Ness DE, Kiesling SF, Lidz CW. Why does informed consent fail? A discourse analytic approach. J Am Acad Psychiatry Law 2009;37:349-362.

35. Miller FG, Rosenstein DL. The therapeutic orientation to clinical trials. N Engl J Med 2003;348:1383-1386.

36. Kimmelman J, Palmour N. Therapeutic optimism in the consent forms of phase 1 gene transfer trials: an empirical analysis. J Med Ethics 2005;31:209-214.

37. Faden RR, Feinberg KR, Oleinick NL, Glatstein E, Royal $\mathrm{HD}$, Katz J, et al. Advisory committee on human radiation experiments: final report. Washington DC:Joseph Henry Press; 1995.

38. Gotay CC. Accrual to cancer clinical trials: directions from the research literature. Soc Sci Med 1991;33:569-577.

39. Schaeffer MH, Krantz DS, Wichman A, Masur H, Reed E, Vinicky JK. The impact of disease severity on the informed consent process in clinical research. Am J Med 1996;100: 261-268.

40. Easter MM, Henderson GE, Davis AM, Churchill LR, King NM. The many meanings of care in clinical research. Sociol Health Illn 2006;28:695-712.

41. Brody JL, Scherer DG, Annett RD, Turner C, Dalen J. Family and physician influence on asthma research participation decisions for adolescents: the effects of adolescent gender and research risk. Pediatrics 2006;118:e356-e362.

42. de Melo-Martín I, Ho A. Beyond informed consent: the therapeutic misconception and trust. J Med Ethics 2008; 34:202-205.

43. Angst DB, Hill JM. Clinical trials for children: in whose best interest? Bull Park Ridge Cent 2000;(18):9-10.

44. Weinfurt KP, Sulmasy DP, Schulman KA, Meropol NJ. Patient expectations of benefit from phase I clinical trials: linguistic considerations in diagnosing a therapeutic misconception. Theor Med Bioeth 2003;24:329-344.

45. Fisher CB, Cea CD, Davidson PW, Fried AL. Capacity of persons with mental retardation to consent to participate in randomized clinical trials. Am J Psychiatry 2006;163:18131820.

46. Miller FG, Wendler D. The relevance of empirical research in bioethics. Schizophr Bull 2006;32:37-41.

47. Sobel D. Sleep study leaves subject feeling angry and confused. The New York Times. Jul 17, 1980;Sect C. p.3C.

48. Wennberg JE, Fisher ES, Goodman DC, Skinner JS. Tracking the care of patients with severe chronic illness: The Dartmouth atlas of health care 2008. Lebanon, NH:The Dartmouth Institute for Health Policy and Clinical Practice; 2008.

49. Pace C, Miller FG, Danis M. Enrolling the uninsured in clinical trials: an ethical perspective. Crit Care Med 2003;31(3 Suppl):S121-S125.

50. Elkashef A, Rawson RA, Smith E, Pearce V, Flammino F, Campbell J, et al. The NIDA Methamphetamine Clinical Trials Group: a strategy to increase clinical trials research capacity. Addiction 2007;102 Suppl 1:107-113.

51. Epstein S. Impure science: AIDS, activism, and the politics 
of knowledge. Berkeley:University of California Press;1996.

52. Glannon W. Phase I oncology trials: why the therapeutic misconception will not go away. J Med Ethics 2006;32:252255.

53. Swekoski D, Barnbaum D. The gambler's fallacy, the therapeutic misconception, and unrealistic optimism. IRB 2013; 35:1-6.

54. Kimmelman J. The therapeutic misconception at 25: treatment, research, and confusion. Hastings Cent Rep 2007;37: 36-42.

55. Shatz D. Randomized clinical trials and the problem of suboptimal care: an overview of the controversy. Cancer Invest 1990;8:191-205.

56. Henderson GE, Davis AM, King NM, Easter MM, Zimmer $\mathrm{CR}$, Rothschild $\mathrm{BB}$, et al. Uncertain benefit: investigators' views and communications in early phase gene transfer trials. Mol Ther 2004;10:225-231.

57. Aaronson NK, Visser-Pol E, Leenhouts GH, Muller MJ, van der Schot AC, van Dam FS, et al. Telephone-based nursing intervention improves the effectiveness of the informed consent process in cancer clinical trials. J Clin Oncol 1996; 14:984-996.

58. Benson PR, Roth LH, Appelbaum PS, Lidz CW, Winslade WJ. Information disclosure, subject understanding, and informed consent in psychiatric research. Law Hum Behav 1988; 12:455-475.

59. Simes RJ, Tattersall MH, Coates AS, Raghavan D, Solomon HJ, Smartt H. Randomised comparison of procedures for obtaining informed consent in clinical trials of treatment for cancer. Br Med J (Clin Res Ed) 1986;293:1065-1068.

60. Annas GJ, Grodin MA. The Nazi doctors and the Nuremberg code: Human rights in human experimentation. New York: Oxford University Press; 1992.

61. Denise Diianni (director), Films for the Humanities (firm); WGBH (television station). The deadly deception [documentary]. Boston, MA:Nova Production and WGBH Educational Foundation; 1993.

62. Hornblum AM. Acres of skin: Human experiments at
Holmesburg prison. New York:Routledge;1998.

63. Lifton RJ. The Nazi doctors: Medical killing and the psychology of genocide. New York:Basic Books;2000.

64. Cunningham PJ. Beyond parity: primary care physicians' perspectives on access to mental health care. Health Aff (Millwood) 2009;28:w490-w501.

65. Miller M. Phase I cancer trials. A collusion of misunderstanding. Hastings Cent Rep 2000;30:34-43.

66. Bankert EA, Amdur RJ. Institutional review board: management and function. Sudbury, MA:Jones and Bartlett Publishers; 2006.

67. Klitzman RL. How IRBs view and make decisions about consent forms. J Empir Res Hum Res Ethics 2013;8:8-19.

68. Perlman D. Ethics in clinical research: A history of human subject protections and practical implementation of ethical standards [Internet]. Chalfont, PA: SoCRA SOURCE; 2004 May [cited at 2015 Aug 3]; 37-40. Available from: http:// materiais.dbio.uevora.pt/MA/Modulo2/Artigos/SoCRA-Perlman. $p d \mathrm{f}$

69. Featherstone K, Donovan JL. Random allocation or allocation at random? Patients' perspectives of participation in a randomised controlled trial. BMJ 1998;317:1177-1180.

70. Waggoner WC, Mayo DM. Who understands? A survey of 25 words or phrases commonly used in proposed clinical research consent forms. IRB 1995;17:6-9.

71. Matutina RE. The concept analysis of therapeutic misconception. Nurse Res 2010;17:83-90.

72. Shore D. Ethical issues in schizophrenia research: a commentary on some current concerns. Schizophr Bull 2006; 32:26-29.

73. Meurer WJ, Lewis RJ, Berry DA. Adaptive clinical trials: a partial remedy for the therapeutic misconception? JAMA 2012;307:2377-2378.

74. Dresser R. The ubiquity and utility of the therapeutic misconception. Soc Philos Policy 2002;19:271-294.

75. Miller FG, Brody H. A critique of clinical equipoise. Therapeutic misconception in the ethics of clinical trials. Hastings Cent Rep 2003;33:19-28. 